\title{
Observation of Optical Undular Bores in Multiple Four-Wave Mixing
}

\author{
J. Fatome, ${ }^{1, *}$ C. Finot, ${ }^{1}$ G. Millot, ${ }^{1}$ A. Armaroli, ${ }^{2}$ and S. Trillo ${ }^{3, \dagger}$ \\ ${ }^{1}$ Laboratoire Interdisciplinaire Carnot de Bourgogne, UMR 6303 CNRS-Université de Bourgogne, \\ 9 Avenue A. Savary, 21078 Dijon, France \\ ${ }^{2}$ Max Planck Institute for the Science of Light, Günther-Scharowsky-Strasse 1/Bau 24, \\ 91058 Erlangen, Germany \\ ${ }^{3}$ Department of Engineering, University of Ferrara, via Saragat 1, 44122 Ferrara, Italy \\ (Received 17 January 2014; revised manuscript received 12 March 2014; published 5 May 2014)
}

\begin{abstract}
We demonstrate that wave-breaking dramatically affects the dynamics of nonlinear frequency conversion processes that operate in the regime of high efficiency (strong multiple four-wave mixing). In particular, by exploiting an all-optical-fiber platform, we show that input modulations propagating in standard telecom fibers in the regime of weak normal dispersion lead to the formation of undular bores (dispersive shock waves) that mimic the typical behavior of dispersive hydrodynamics exhibited, e.g., by gravity waves and tidal bores. Thanks to the nonpulsed nature of the beat signal employed in our experiment, we are able to clearly observe how the periodic nature of the input modulation forces adjacent undular bores to collide elastically.
\end{abstract}

\section{INTRODUCTION}

Under certain regimes, the behavior of light mimics the dynamics of classical fluids and, in particular, hydrodynamical phenomena [1-15]. The general ground for such observation lies on the reduction of Maxwell equations to the typical models of hydrodynamics, as recognized long ago for both dissipative (i.e., lasers [1,2]) and conservative settings [3]. Only recently, however, has the subject attracted a great deal of interest, propelling important experimental results as diverse as the first absolute observation of wave packets predicted in hydrodynamics (i.e., Peregrine and Kuznetsov-Ma solitons [6,7]), the study of classical hydrodynamical instabilities (e.g., Raileigh-Taylor [8]), the characterization of extreme events such as rogue waves or tsunamis [9-12], the flow of quantum fluid of light around defects at low and supersonic speed [13], or the transition to turbulence in fiber lasers [14]. On one hand, this allows us to improve the understanding of fluid phenomena by means of accurate and more easily controllable lab experiments; on the other hand, it also stimulates the transposal to hydrodynamics of concepts, e.g., generations of higher-order breathers and supercontinuum [16-18], that are deeply studied in optics $[19,20]$.

\footnotetext{
jfatome@u-bourgogne.fr

†stefano.trillo@unife.it
}

Published by the American Physical Society under the terms of the Creative Commons Attribution 3.0 License. Further distribution of this work must maintain attribution to the author(s) and the published article's title, journal citation, and DOI.
In this framework, another remarkable phenomenon that allows for establishing an intriguing photon-fluid analogy, which is of concern in this paper, is the generation of undular bores (UBs), i.e., the formation of fast oscillating wave trains that spontaneously emerge from points of gradient catastrophe (shock formation [21]). In hydrodynamics, UB are fascinating structures that develop under peculiar conditions involving, e.g., atmospheric gravity waves (e.g., "Morning glory" phenomena [22]), internal waves in the ocean [23] or, more commonly, large tidal bores traveling upstream, typically along river estuaries [24]. A spectacular and famous example of the latter (among others visible in other parts of the world [24]) is the so-called "mascaret" of the Dordogne river in France, ridden by many surfers. The study of such phenomena started indeed long ago in hydrodynamics [25] and independently in the context of strongly rarified (i.e., collisionless) plasma, where such structures were known as collisionless shock waves [26-29]. Such studies established dispersion as the driving mechanism that, by regularizing the steep front characteristic of a classical shock wave, leads to UBs (hence also the expression "dispersive shock waves"). While the theoretical characterization of such phenomena has improved steadily [30-40], the field has suffered the lack of reproducible lab experiments, until the universal role of UB was recently recognized mainly in the dynamics of quantum superfluids [41-47] and weakly diffracting light beams in defocusing media $[4,5,48-52]$, both described by defocusing nonlinear Schrödinger (NLS, or Gross-Pitaevskii) models. At variance with such experiments where undulatory breaking occurs in space, optics also offers the challenging 
possibility to investigate temporal wave breaking, as realized for intense (hundreds of Watts peak power) pulses that propagate in optical fibers in the normal group-velocity (GVD) regime [53-56]. In this context, the aim of this paper is twofold. First, we report evidence that temporal breaking and emerging UBs affect a completely novel realm, namely, frequency conversion processes, and specifically highly efficient multiple four-wave mixing (FWM) [57-60], under different pump configurations. Second, we show that the wave-breaking regime is accessible, in an allfiber platform without resorting to intense (psec or fsec) pulses, using instead a tailorable, nearly periodic signal. The latter feature is the key to our first observation of postbreaking multiphase dynamics characterized by unavoidable collision of UBs due to the periodic nature of the input.

More generally, optical FWM is of great interest not only because it is the fundamental paradigm for parametric amplification and generation, but also because it is widely exploitable in different materials and platforms including planar (on-chip) devices [61-65], and it is the driving mechanism of more complex phenomena such as supercontinuum generation and wave thermalization $[19,20]$. Moreover, FWM has made possible a host of modern photonic applications ranging from ultrafast pulse train generation [66], full signal regeneration [67], multiwavelength parametric oscillators [68], stable ultrafast microcavity lasers [69], and bridging of spectral windows [65], to recent breakthroughs such as the implementation of noiseless amplifiers for ultrasensitive links [70] and frequency combs [71-74]. Along with these achievements, more fundamental aspects related to the spectral dynamics of FWM have also been the subject of recent studies $[60,73,75]$. In this context, the problem of wave breaking, which has never been addressed experimentally, is of particular interest since it can affect the FWM in the regime of weak normal dispersion, which is often exploited to obtain several multiple signal-idler sideband pairs at once [73-78]. Here, we report extensive evidence that, in this regime, FWM unavoidably enters a new scenario in which the initial modulation undergoes a hydrodynamic type of instability that deeply affects the mixing process. The light field indeed behaves as a fluid, though it exhibits two distinct stages of evolution: the first featuring the developments of gradient catastrophes typical of shallowwater dynamics, followed by the onset of fast oscillations characteristic of UBs of dispersive hydrodynamics.

\section{UNDULAR BORES IN FOUR-WAVE MIXING}

Two laser pumps (or a single modulated pump) produce, via Kerr (cubic) nonlinearities, a frequency cascade via multiple FWM, whose efficiency can be easily calculated in the limit of zero dispersion [78]. However, even a small dispersion can qualitatively change the FWM dynamics $[60,73]$. Of particular interest is the normal GVD regime which, since it is not affected by exponentially growing perturbations (i.e., modulational instability $[75,79]$ ), exhibits a fluidlike shock-forming type of behavior in the regime of propagation dominated by Kerr nonlinearity over dispersion, as recently predicted in the case of a dual pump [60]. Here, however, we considerably generalize such a theory by showing that the phenomenon is not restricted to dual-frequency input. Indeed, any initial amplitude modulation tends to break when FWM operates under weak, normal GVD. Specifically, we can treat, in a unified way, a modulated carrier (triple-frequency input with pulsations $\omega_{0}, \omega_{0} \pm \Delta \omega$ ), as well as a suppressed carrier or dual-frequency input at $\omega_{0} \pm \Delta \omega / 2$ (balanced dual-pump nondegenerate FWM or unbalanced pump-signal configuration). Starting from the real-world input envelope at central frequency $\omega_{0}$, say, $A(Z=0, T)=$ $\sqrt{P} u_{0}(t)$, which is modulated at frequency $\Delta f=\Delta \omega / 2 \pi$ and carries total power $P\left[u_{0}(t)\right.$ is a dimensionless envelope carrying unit power], these three configurations can be described by the following general expression for the input

$$
u_{0}(t)=\sqrt{\eta}+\sqrt{\eta_{+}} \exp (i \Omega t / m)+\sqrt{\eta_{-}} \exp (-i \Omega t / m)
$$

where, without loss of generality, the normalized time $t=$ $T / T_{0}$ is measured in units of the characteristic rise time of the modulation $T_{0}=1 / 2 \Delta f$, which amounts to keeping the normalized angular frequency $\Omega=\Delta \omega T_{0}=\pi$ fixed. $\eta$ and $\eta_{ \pm}=(1-\eta \pm \alpha) / 2$ stand for power fractions at discrete frequencies $\omega_{0}$ and $\omega_{0} \pm \Delta \omega / m$, respectively, with constraint $\eta+\eta_{+}+\eta_{-}=1$, while $\alpha \equiv \eta_{+}-\eta_{-}$defines the input power imbalance. Here and in Eq. (1), $m=1$ or $m=2$ for the triple-frequency $(\eta \neq 0)$ or dual-frequency $(\eta=0)$ input, respectively, so that $\Delta \omega$ designates the detuning between the input angular frequencies in all three cases [see also the sketch in Fig. 1(a)].

When dispersion is weak, the modulated field produces a FWM cascade, i.e., generation of harmonic sidebands at normalized angular frequencies $\pm n \Omega / 2(n=4,6,8 \ldots$ with triple-frequency input and $n=3,5,7 \ldots$ for dual-frequency input), which is described in terms of the normalized envelope $u(z, t)$ obeying the following NLS equation (see Ref. [60] and also Ref. [80] for more details on the normalization):

$$
i \varepsilon \frac{\partial u}{\partial z}-\frac{\varepsilon^{2}}{2} \frac{\partial^{2} u}{\partial t^{2}}+|u|^{2} u=0
$$

where $z=Z \sqrt{k^{\prime \prime} \gamma P} / T_{0}$ is the normalized distance, and it turns out that the dynamics depends on the single parameter $\varepsilon \equiv \Delta f \sqrt{4 k^{\prime \prime} / \gamma P}$, where $k^{\prime \prime}=d^{2} k / d \omega^{2}$ and $\gamma$ denote the GVD and nonlinear coefficient of the fiber, respectively.

Our experiments address the weakly dispersive regime of FWM characterized by $\varepsilon \ll 1(\varepsilon=0.04$ at the maximum power reached in the experiment). In this regime, the light field behaves as an ideal equivalent fluid or gas, where the 

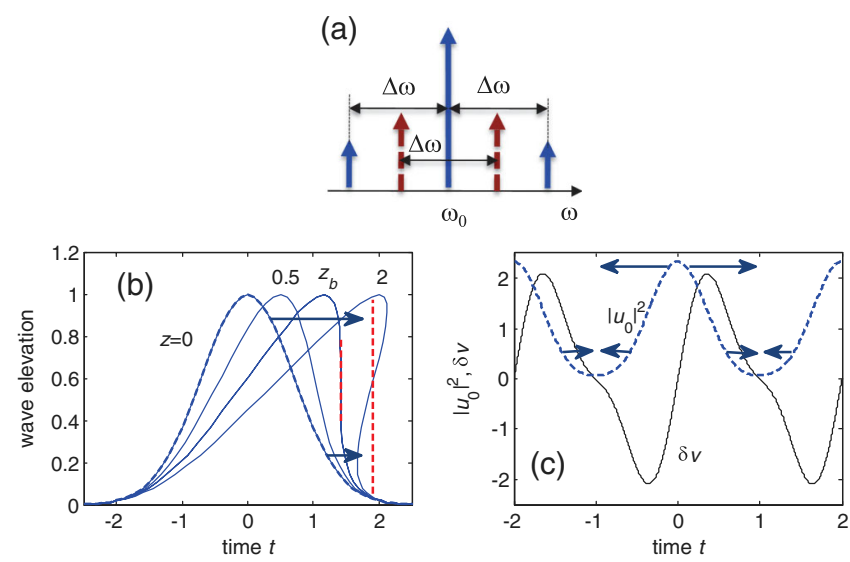

FIG. 1. (a) Sketch of the input in frequency domain showing the triple-frequency (solid blue arrows) and dual-frequency (dashed brown arrows) cases, respectively. (b) Generic mechanism of wave breaking via a gradient catastrophe (length of the arrows is proportional to the local velocity at the point of application over the input; the dashed vertical line is the classical shock wave). (c) Initial power profile $\left|u_{0}(t)\right|^{2}$ and instantaneous incremental velocity $\delta v(t)$ resulting from the nonlinear selfphase modulation for triple-frequency input $[\eta=0.8, \alpha=0$, $m=1$ in Eq. (1)].

smooth input modulation undergoes strong steepening driven by the Kerr effect (cubic term in NLS equation [81]), eventually forming shock waves through a gradient catastrophe. The latter is a universal mechanism that occurs whenever the wave velocity becomes a function of the wave elevation, as illustrated in Fig. 1(b) for a real field $u(z, t)$ obeying the prototypical Hopf or inviscid Burger equation $u_{z}+u u_{t}=0$. [Here, we implicitly assume that $t$ stands for a retarded time in a frame moving at linear group velocity $v_{g}$ as in the NLS equation (2). This means that the deformation illustrated in Fig. 1(b) takes place, in the lab frame, on top of a rigid translation with linear velocity $v_{g}$ ]. Since this model implies that portions of a waveform with larger elevations move faster, a smooth envelope such as, e.g., a Gaussian is deformed on propagation as shown in Fig. 1(b), until it develops an infinite time gradient (gradient catastrophe) at a finite distance $z_{b}$, and a traveling vertical front afterwards. The latter is a classical shock wave that normalizes the post-catastrophe overtaking while obeying the integral conservation law associated with the differential model [21].

A similar mechanism, as we show here, occurs in FWM, owing to the fact that the input field $u_{0}(t)$ develops a selfphase modulation that stems from the dominant nonlinear term in Eq. (2). The acquired phase is proportional to $\left|u_{0}(t)\right|^{2}$, in turn implying an instantaneous frequency deviation (chirp) $\delta \omega(t)=\partial_{t} \phi(t)=\partial_{t}\left|u_{0}(t)\right|^{2}$ [54]. Such a chirp is turned into an instantaneous change of velocity $\delta v(t) \propto-\delta \omega(t)$ since in a normally dispersive medium the velocity decreases for increasing frequency. As a result, the instantaneous velocity turns out to be directed along the outward direction around the maxima and along the inward directions around the minima of the modulation, as exemplified in Fig. 1(c) for the triple-frequency modulated input with $\eta=0.8, \alpha=0$. Therefore, compressional waves are created that tend to steepen the initially smooth modulation fronts around the intensity minima. In turn, the steepening causes an additional increase in the modulus of the opposite velocities across the minima, thus producing further steepening, until the process leads to gradient catastrophes at a finite distance. Formally, this can be seen by applying the Wentzel-Kramers-Brillouin (WKB) or Madelung transformation $u(z, t)=\sqrt{\rho(z, t)} \exp \left[\frac{i}{\varepsilon} s(z, t)\right]$ to Eq. (2), which allows us to map the original NLS equation into the following quasilinear system of equations [3],

$$
\rho_{z}+(\rho v)_{t}=0 ; \quad v_{z}+v v_{t}+\rho_{t}=0
$$

(a)

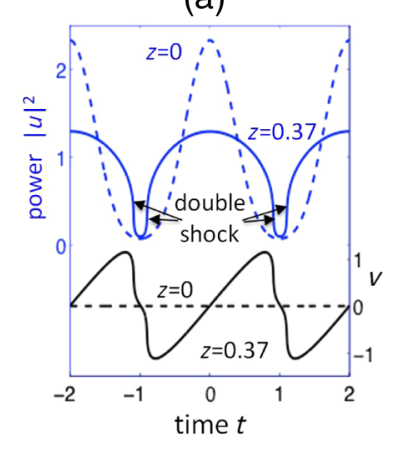

(b)

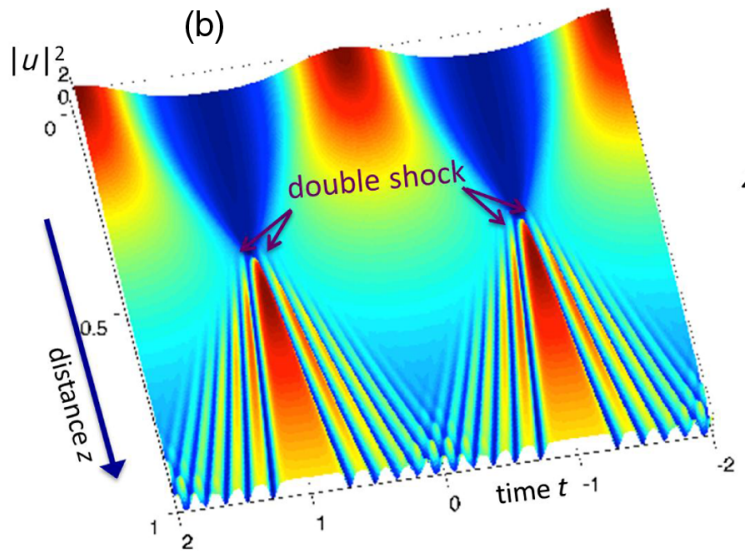

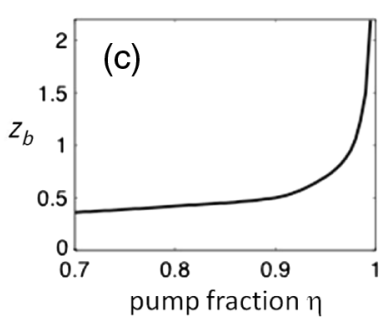

FIG. 2. Breaking mechanism for an input modulated carrier (three-wave input, $\eta=0.8$ and $\alpha=0$ ). (a) Normalized power $\rho=|u|^{2}$ and velocity $v$ at wave-breaking distance $z=z_{b} \simeq 0.37$ from the reduced hydrodynamical model [Eqs. (3)]; the dashed curves stand for the input. (b) Surface plot of power $|u(z, t)|^{2}$ evolving according to the NLS equation [Eq. (2)] for $\varepsilon=0.04$. (c) Normalized breaking distance $z_{b}$ vs pump power fraction $\eta$. 
where terms of higher orders in power of $\varepsilon$ have been discarded. Equations (3) are equations of the hydrodynamic type, which can be integrated, up to the point where a singularity appears, by means of the so-called hodograph transformation [21]. Indeed, Eqs. (3) are identical to those governing 1D shallow-water waves (or isentropic gas dynamics), with the roles of space and time inverted, and $\rho(z, t)=|u(z, t)|^{2}$ and $v(z, t)=-\partial_{t} s(z, t)$ being the water elevation and horizontal velocity, respectively. According to this model, the light behaves as an equivalent fluid whose instantaneous velocity is determined by the accumulated chirp. The evolution of the fluid leads to the formation of an array of twin shock points symmetrically located around the minima of the input modulation, as shown in Fig. 2(a), obtained by numerical integration of Eqs. (3). We point out that this mechanism takes place regardless of the modulation depth, though the breaking distance $z_{b}$ increases rapidly as the modulation depth decreases $(\eta \rightarrow 1)$, as shown in Fig. 2(c). Importantly, however, beyond $z_{b}$, Eqs. (3) cease to be valid because the GVD becomes important near the vertical fronts, causing the onset of fast nonstationary oscillations that fill characteristic shock fans. This is displayed in Fig. 2(b), which is obtained by numerical integration of the NLS equation. As shown, these structures, namely, UBs (or dispersive shock waves), naturally collide because of the periodic nature of the input. A similar dynamics also takes place for the carrier-suppressed modulation (two-wave input, $\eta=0$ ), which still leads to two distinct points of breaking for each period of the modulation in the imbalanced case $(\alpha \neq 0)$. These points merge exactly in the null points of the modulation in the limit $\alpha=0$, as discussed in more detail in Ref. [60].

\section{EXPERIMENTAL IMPLEMENTATION}

Our experiment was carefully designed in order to succeed with two major challenges raised by the observation of the phenomenon discussed above: (i) to access the highly nonlinear regime without using pulses, which would unavoidably corrupt the visibility of the UBs; (ii) to reproduce the evolution dynamics using a fixed fiber length. To this end, in our setup, displayed in Fig. 3, we make use of an $L=6 \mathrm{~km}$-long nonzero dispersionshifted fiber (NZDSF) with low normal dispersion $D=$ $-2.5 \mathrm{ps} / \mathrm{nm} \mathrm{km} \quad\left(k^{\prime \prime}=-D \lambda_{0}^{2} / 2 \pi c=3.2 \mathrm{ps}^{2} / \mathrm{km}\right) \quad$ and nonlinear coefficient $\gamma=1.7 \mathrm{~W}^{-1} \mathrm{~km}^{-1}$. We point out that the choice of this value of dispersion arises from a tradeoff between opposite requirements. On the one hand, the GVD must be sufficiently low to enter the weakly dispersive regime at the central frequency and at the power levels involved in the experiment, while on the other hand, the GVD needs to be large enough so that third-order dispersion (as well as successive orders) remains negligible. We inject the field $A(0, T)=\sqrt{P} u_{0}(t)$, which is obtained by starting from an external cavity laser (ECL) emitting at wavelength $\lambda_{0}=1555 \mathrm{~nm}$, modulated by means of a $\mathrm{LiNbO}_{3}$ intensity modulator (IM\#1) at $\Delta f=28 \mathrm{GHz}$. By suitably driving the modulator, we can select a dual-frequency (carrier-suppressed) or threefrequency input modulation, depending on its bias operating point (see Ref. [80] for further technical details). The peak power is then raised by means of further slicing in the time domain (using modulator IM\#2) and amplification in an erbium-doped fiber amplifier (EDFA). The final input signal consists of a burst of eight periods of the initial 28-GHz harmonic signal encapsulated into a 1:4 dutycycle slower signal at 7-GHz (see inset in Fig. 3), which allows us to reach the power $P=37 \mathrm{dBm}(P \simeq 5 \mathrm{~W})$ without a significant impact from the spontaneous emission of the EDFA and stimulated Brillouin backscattering within the fiber under test. It is important to emphasize that the eight-beats sequence is long enough to ensure that the observed dynamics is, at least over the central periods of the sequence, essentially that of the temporal infinite beat signal. At the output, the temporal ultrafast waveform can be observed directly over a triggered optical sampling oscilloscope (OSO). In Fig. 3, we display traces of the input and the output in the regime of UB formation, as seen on the OSO display. Importantly, even if we use a fixed fiber length $L$, the spatial dynamics along $z$ is mimicked by varying the input power $P$, recalling that $z=L \sqrt{k^{\prime \prime} \gamma P} / T_{0}$.

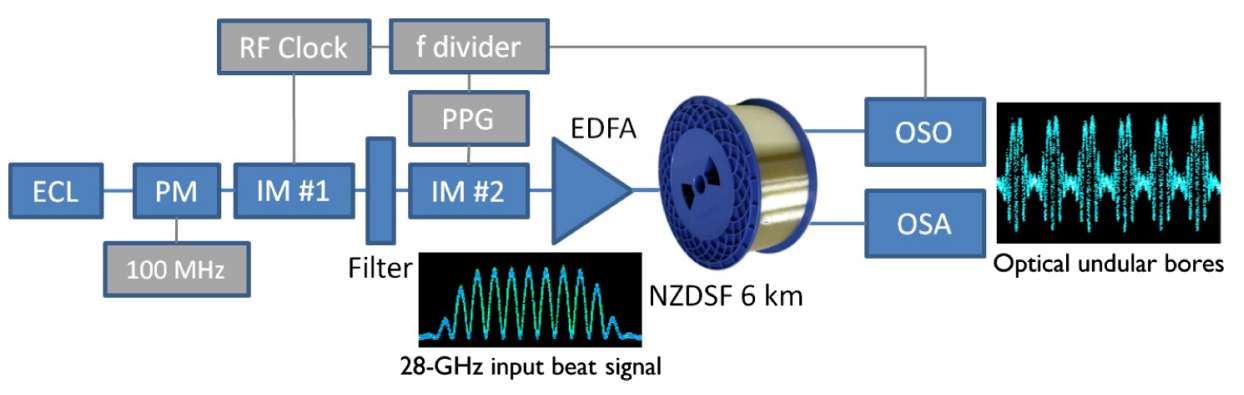

FIG. 3. Experimental setup. ECL: external cavity laser; IM: intensity modulators; PM: phase modulator; PPG: pulse pattern generator; OSO: optical sampling oscilloscope; OSA: optical spectrum analyzer; NZDSF: nonzero dispersion shifted fiber. The insets show traces of the input and output, as seen on the OSO. 


\section{EXPERIMENTAL RESULTS}

The outcome of the experiments is illustrated in Figs. 4-6 under different input configurations of FWM. Figure 4 summarizes the results obtained for the case of balanced dual-frequency input (symmetric pump beams, $\eta=\alpha=0$ ). The experimental maps of the temporal dynamics vs input power, reported both as a 3D surface [Fig. 4(a)] and a colorlevel plot [Fig. 4(b)], clearly show the signature of UBs in the evolution. Furthermore, the measured data are in excellent quantitative agreement with the outcome of the numerical integration of the NLS equation reported in Fig. 4(c), without any adjustable parameter. Note that such results are obtained from a set of numerical simulations of the NLS equation in dimensional units [80], performed at fixed physical length and variable power. In this configuration, the input modulation is a cosine, and breaking is expected through the formation of cusplike structures exactly at the nulls of the input intensity pattern [60]. We observe such behavior at the power level $P=25.6 \mathrm{dBm}$, as shown explicitly by the OSO trace reported in Fig. 4(d). After breaking, strong oscillations characteristic of UBs start to appear on a faster scale (few ps) compared with the input periodicity $T_{m}=1 / \Delta f=35.7 \mathrm{ps}$. The undular bores are nucleated from the breaking points at nulls and expand outwards in a nonstationary and symmetric fashion until wave trains from adjacent periods of the input beat signal start to collide, as clearly shown also by the trace in Fig. 4(e). We remark that the temporal structure of the overlapping regions can be clearly observed, thanks to the nearly periodic nonpulsed nature of the input, since the use of pulses would hamper its visibility, as one can easily verify through numerical simulations of the pulsed regime (not shown). The signature of the fast oscillatory UB structure in the frequency domain is a dramatic spectral broadening. In Fig. 4(f), we compare the output spectrum before breaking (blue trace, $P=13 \mathrm{dBm}$ ) with that arising in the regime where the UB is fully developed (red trace, $P=34.6 \mathrm{dBm}$ ). The latter involves tens of FWM harmonic pairs while presenting significant deviations from the monotonic decay (in $\log$ scale) characteristic of the low-power regime that stems from the high frequencies corresponding to the oscillatory structures. It is also interesting to highlight the fact that in the experiment, the post-breaking undulations clearly exhibit solitonlike features. Indeed, they present negligible temporal spreading while moving with characteristic velocity inversely proportional to their darkness and colliding elastically. The main feature of the optical UB is, in this case, the formation of a still temporal filament possessing the features of a black soliton [the central filament in Figs. 4(b) and 4(c)], which arises from the presence of null
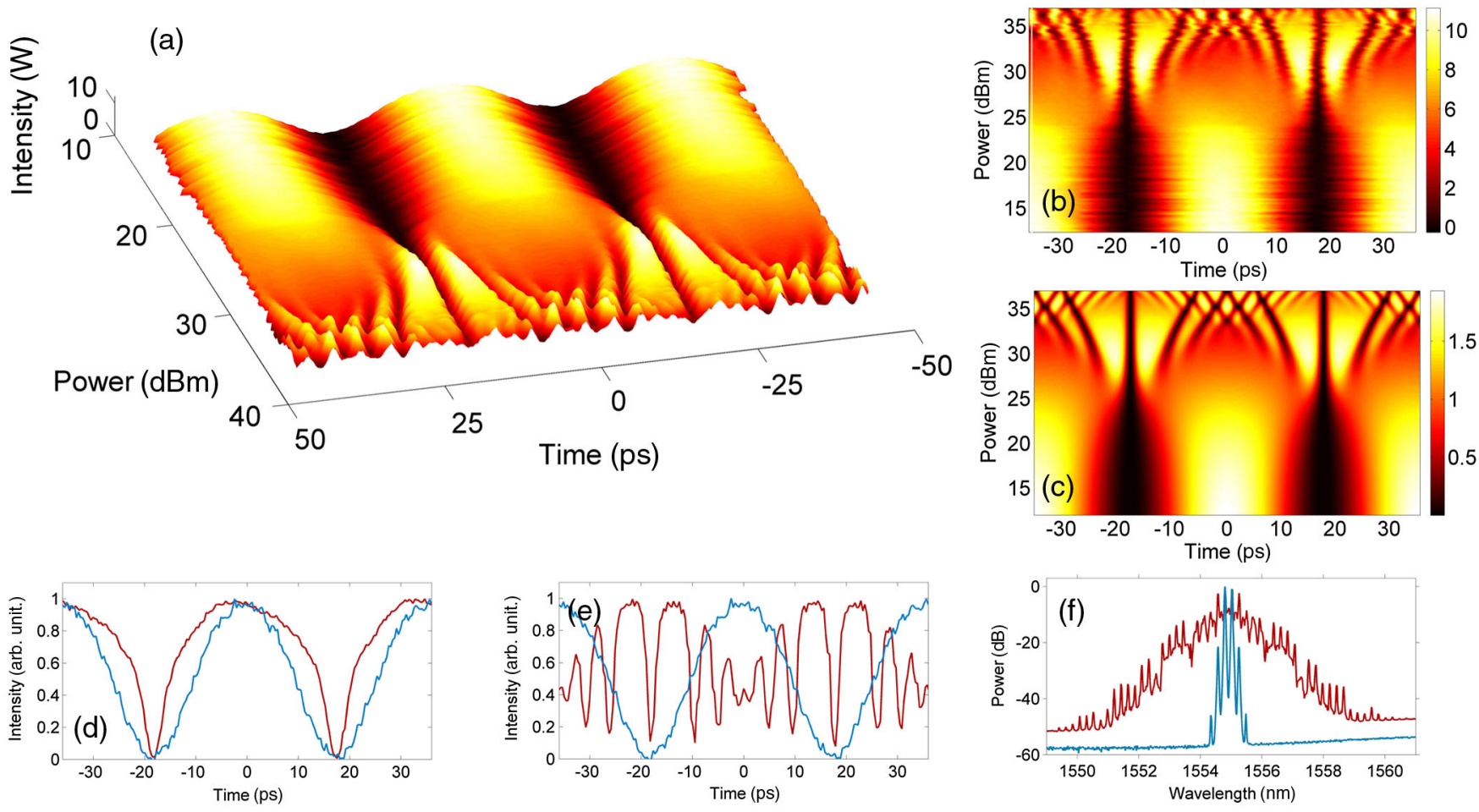

FIG. 4. Measured UB dynamics for symmetric dual-pumped FWM (cosine type of input, $\eta=\alpha=0$ ). (a) 3D surface of output power vs time and input power. (b),(c) Color-level plot from the output power profiles at different input powers from measured data (b), compared with numerical simulations of the dimensional NLS equation [(c), color level plot of $\left.|u|^{2}\right]$. (d), (e) Temporal OSO traces at power levels: (d) $P=25.6 \mathrm{dBm}$, close to breaking; (e) $P=34.6 \mathrm{dBm}$, beginning of UB collision (the input is reported as a solid blue line). (f) Output spectra in the low-power $(P=13 \mathrm{dBm}$, preshock) and high-power $(P=34.6 \mathrm{dBm}$, postshock $)$ regimes. 
intensity points in the initial modulation. Gray solitons with opposite velocity appear in pairs around the latter, progressively filling a characteristic shock fan, at the edges of which the oscillations (solitons) become progressively more shallow. Colliding shock fans form regions of so-called multiphase dynamics [37] characterized by very complicated temporal dependence determined by the overlap of oscillations arising from adjacent UBs. In this respect, the dynamics is completely different from other schemes proposed in the past for the generation of dark soliton trains [34,56] and the related generation of broadband emission [82]. Indeed, in these schemes, the use of powerful pulses is mandatory, and the spectrum is not characterized by a discrete-line (comb) structure. In addition, the asymptotic state tends to a clean train of dark solitons, which is never the case in any of our FWM experiments (see also the discussion in the last paragraph of this section). Importantly, our data show a remarkable degree of symmetry, both temporally [Fig. 4(b)] and spectrally [Fig. 4(f)], giving evidence of the negligible role of third-order dispersion in our experiment. Indeed, it is easy to show that third-order dispersion would induce temporal symmetry breaking (for instance, in this configuration the central filament would acquire a velocity, no longer possessing a zero-intensity dip) and resonant enhancement of a low-frequency part of the spectral FWM comb [73]. The absence of these effects proves that our experiment is accurately described by the NLS equation (2) with no extra term, despite the fact that GVD is weak.

We have also assessed, experimentally, the effect of an input imbalance over the dual-frequency carrier-suppressed configuration, where FWM is first responsible for the generation of the idler (the spectral image of the weaker
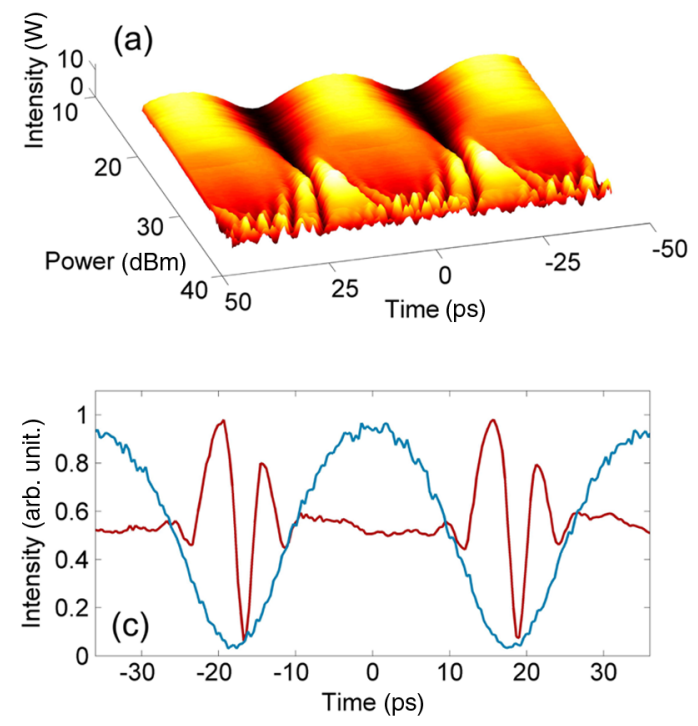

input signal) and then for all the harmonics of the signalidler pair. As shown in Fig. 5, an imbalance $\alpha=0.3$ introduced in the input causes the input waveform to break at two distinct instants (per period) around the intensity minima. From such a pair of breaking points, two asymmetric undular bores emerge which fill shock fans pointing in opposite directions. These fans, arising from adjacent periods, then naturally collide. In this case, the input waveform does not have intensity nulls, and the leadingorder oscillations (inner edges of the fans) are grey solitons that possess different nonzero velocities, as shown in the trace in Fig. 5(c). The data in Fig. 5(b) and the simulations in Fig. 5(d) are in remarkably good agreement in this case, too.

Finally, Fig. 6 illustrates the case of a modulated carrier with symmetric sidebands corresponding to $\eta=0.7$, $\alpha=0$. In this case, breaking occurs, as already illustrated in Fig. 2, at two distinct instants that, in this case, are symmetrically located around the minima of the modulation and occur at the same finite distance. The undular bores reflect the symmetry of the problem, leading to two symmetric shock fans with inner (leading) edges featuring the deepest oscillations. Since the input waveform does not vanish in this case either, these inner edges behave as a pair of gray solitons traveling with opposite (low) velocities. As in previous cases, the fans from adjacent periods of the input modulation naturally collide, as also shown in the snapshot in Fig. 6(c). We point out that, not only do we obtain a close quantitative agreement between the data and the numerics in this case, but the estimated breaking distance $z_{b}=0.36$ from Fig. 2(c) agrees well with the measured value of power for breaking $P=24.5 \mathrm{dBm}$, which corresponds to $z=0.364$.
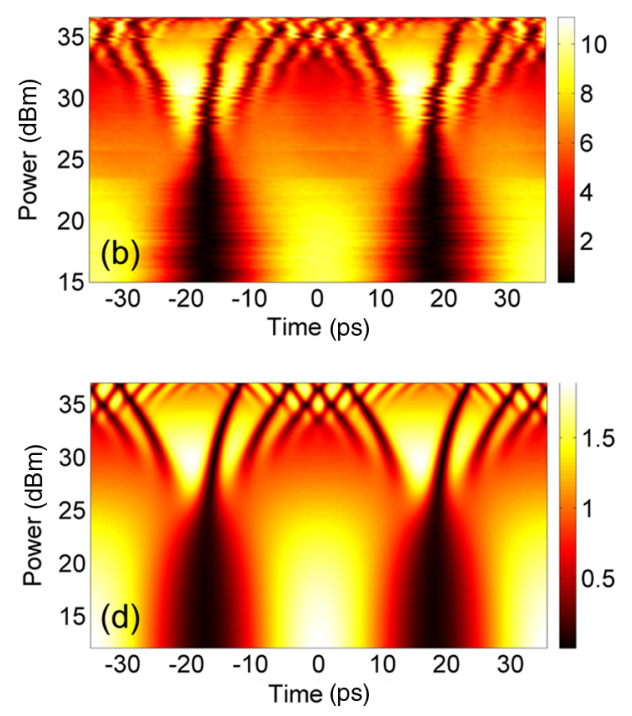

FIG. 5. Measured UB dynamics for the pump-signal input configuration (asymmetric dual-pumped FWM, $\eta=0, \alpha=0.3$ ). (a) 3D surface of measured output power vs time and input power. (b),(d) Color-level plot of the output power profile at different input powers from measured data (b) and numerical simulations of the dimensional NLS equation (d). (c) Temporal OSO trace at $P=30.6 \mathrm{dBm}$, showing the onset of asymmetric oscillations (the solid blue line stands for the input). 

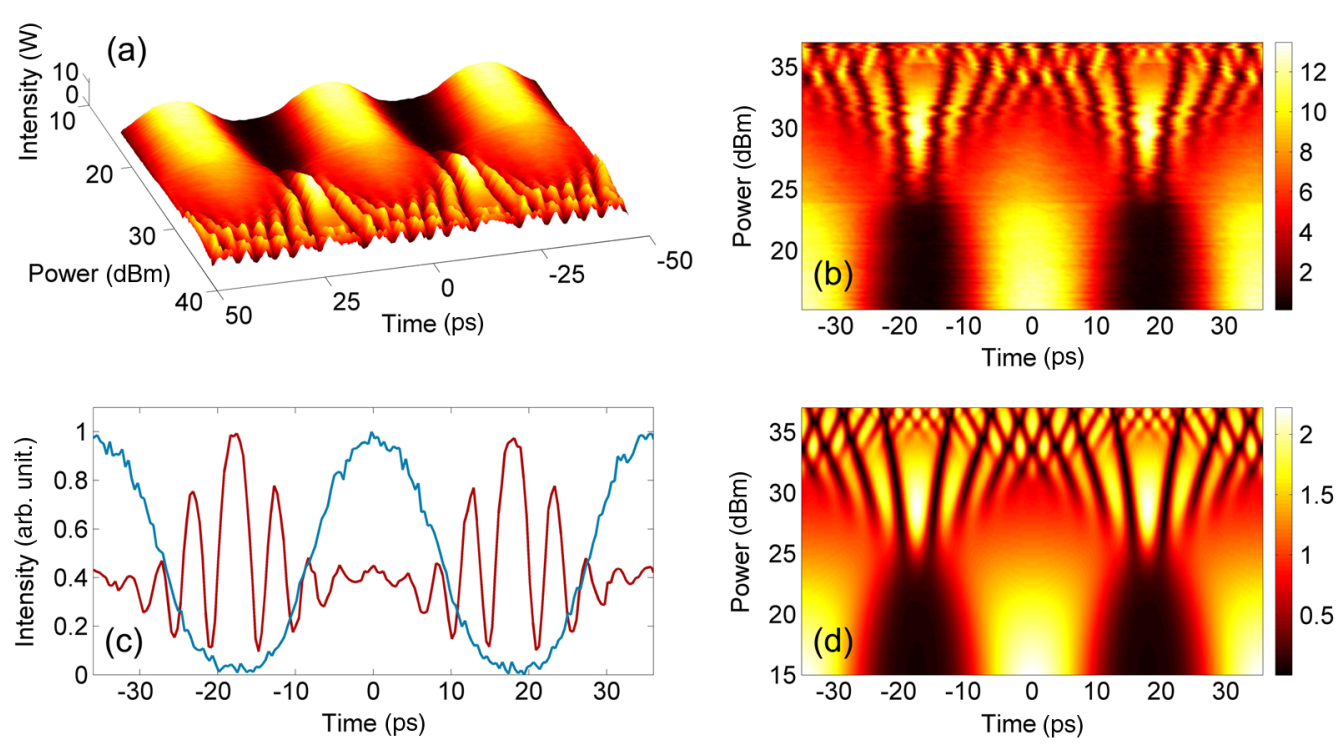

FIG. 6. As in Fig. 5, with FWM produced by the three-wave input with $\eta=0.7$ and $\alpha=0$. The temporal OSO trace in (c) corresponds to a power $P=32 \mathrm{dBm}$.

Before concluding, it is worth specifying in what sense one can talk about solitons with reference to the fast wave trains associated with the UB structure observed in Figs. 4-6. First, it is interesting to note that our experiment, especially in the configuration of Fig. 3 where the input is a cosine, represents the realization, though in the framework of a different model, of the conceptual numerical experiment made in the celebrated paper published nearly 50 years ago by Zabusky and Kruskal [27], which led them to coin the word "solitons" to indicate nonlinear modes nucleated by wave breaking of a sinusoidal input. Generally speaking, however, an input wave packet that undergoes breaking and forms dispersive shocks or UBs cannot be considered to be a soliton phenomenon (with exceptions constituted by specific examples; see, e.g., Ref. [38]). Indeed, UBs also manifest themselves from breaking of waveforms that do not contain any soliton at all (solitons are meant here in the strict sense of integrable models, i.e., discrete eigenvalues of the input potential in the scattering problem associated with the evolution equation). Conversely, UBs are generally described as a nonstationary slow modulation of nonlinear periodic solutions (so-called cnoidal waves) [29]. Specifically, for the case of periodic input waves treated in this paper, the inverse scattering formulation of the NLS equation cannot be made in terms of solitons but rather in terms of so-called finite-band solutions [83]. Nevertheless, the term soliton, which is introduced so as to illustrate the features of the postcatastrophe dynamics in Figs. 4-6, finds, in this context, its justification in the following argument. In the limit $\varepsilon \rightarrow 0$, the number of finite bands diverges while they tend to shrink around their central value, thus reducing to equivalent discrete eigenvalues that are characteristic of solitons on the infinite line [34] (more details on this point, which is evidently an issue of theoretical interest, will be given in a future publication).

\section{CONCLUSIONS}

Thanks to the transposition based on the light-fluid analogy, all-fiber platforms allow the scientific community to perform repeatable experiments on striking and universal-wave phenomena whose exploration in large-scale hydrodynamics remains, to date, extremely challenging, at the same time revealing novel regimes of light propagation. In this contribution, we have focused on the first absolute demonstration of spontaneous temporal wave breaking occurring in the most typical and exploited frequency-conversion processes. More precisely, by means of a specifically designed fiber experiment, we have reported the direct observation of the generation of multiple optical dispersive shocks or undular bores and their natural interactions, under different four-wave mixing configurations. These results thus provide a better understanding of the rich scenarios offered by FWM interactions, especially in connection with the several applications that make use of FWM in the high-efficiency regime. Furthermore, at a fundamental level, our experiment can have a deep impact on the ability to understand dispersive shock waves. These are indeed fascinating and ubiquitous phenomena, though generally challenging to reproduce and characterize in their original environment, as their long-standing history suggests.

\section{ACKNOWLEDGMENTS}

All the experiments were performed on the PICASSO platform in ICB. The research leading to these results has received funding from the European Research Council under the European Community Seventh Framework Programme (FP7 Grant Agreement No. 306633, PETAL project). We also acknowledge the financial support from the Conseil Regional de Bourgogne: Photcom PARI 
program, the Labex ACTION program (Contract No. ANR11-LABX-0001-01) and iXCore Foundation. S. T. acknowledge hospitality by University of Bourgogne and partial support by Italian Ministry of Education, University and Research (MIUR) under Project No. PRIN 2009P3K72Z.

[1] M. Brambilla, L. A. Lugiato, V. Penna, F. Prati, C. Tamm, and C. O. Weiss, Transverse Laser Patterns. II. Variational Principle for Pattern Selection, Spatial Multistability, and Laser Hydrodynamics, Phys. Rev. A 43, 5114 (1991).

[2] K. Staliunas, Laser Ginzburg-Landau Equation and Laser Hydrodynamics, Phys. Rev. A 48, 1573 (1993).

[3] Y. Kodama and S. Wabnitz, Analytical Theory of GuidingCenter Nonreturn-to-Zero and Return-to-Zero Signal Transmission in Normally Dispersive Nonlinear Optical Fibers, Opt. Lett. 20, 2291 (1995).

[4] W. Wan, S. Jia, and J. W. Fleischer, Dispersive Superfluidlike Shock Waves in Nonlinear Optics, Nat. Phys. 3, 46 (2006).

[5] N. Ghofraniha, C. Conti, G. Ruocco, and S. Trillo, Shocks in Nonlocal Media, Phys. Rev. Lett. 99, 043903 (2007).

[6] B. Kibler, J. Fatome, C. Finot, G. Millot, F. Dias, G. Genty, N. Akhmediev, and J. M. Dudley, The Peregrine Soliton in Nonlinear Fibre Optics, Nat. Phys. 6, 790 (2010).

[7] B. Kibler, J. Fatome, C. Finot, G. Millot, G. Genty, B. Wetzel, N. Akhmediev, F. Dias, and J. M. Dudley, Observation of Kuznetsov-Ma Soliton Dynamics in Optical Fibre, Sci. Rep. 2, 463 (2012).

[8] S. Jia, M. Haataja, and J. W. Fleischer, Rayleigh-Taylor Instability in Nonlinear Schrödinger Flow, New J. Phys. 14, 075009 (2012).

[9] K. Hammani, B. Kibler, J. Fatome, S. Boscolo, G. Genty, J. M. Dudley, and C. Finot, Nonlinear Spectral Shaping and Optical Rogue Events in Fiber-Based Systems, Opt. Fiber Technol. 18, 248 (2012).

[10] M. Onorato, S. Residori, U. Bortolozzo, A. Montinad, and F. T. Arecchi, Rogue Waves and Their Generating Mechanisms in Different Physical Contexts, Phys. Rep. 528, 47 (2013).

[11] S. Wabnitz, Optical Tsunamis: Shoaling of Shallow Water Rogue Waves in Nonlinear Fibers with Normal Dispersion, J. Opt. 15, 064002 (2013).

[12] B. Varlot, S. Wabnitz, J. Fatome, G. Millot, and C. Finot, Experimental Generation of Optical Flaticon Pulses, Opt. Lett. 38, 3899 (2013).

[13] I. Carusotto and C. Ciuti, Quantum Fluids of Light, Rev. Mod. Phys. 85, 299 (2013).

[14] E. G. Turitsyna, S. V. Smirnov, S. Sugavanam, N. Tarasov, X. Shu, S. A. Babin, E. V. Podivilov, D. V. Churkin, G. Falkovich, and S. K. Turitsyn, The Laminar-turbulent Transition in a Fibre Laser, Nat. Photonics 7, 783 (2013); E. G. Turitsyna, G. E. Falkovich, V. K. Mezentsev, and S. K. Turitsyn, Optical Turbulence and Spectral Condensate in Long-Fiber Lasers, Phys. Rev. A 80, 031804R (2009).

[15] Editorial, The Power of Analogies, Nat. Photonics 8, 1 (2014).
[16] A. Chabchoub, N. Hoffmann, M. Onorato, and N. Akhmediev, Super Rogue Waves: Observation of a Higher-Order Breather in Water Waves, Phys. Rev. X 2 , 011015 (2012).

[17] A. Chabchoub, N. Hoffmann, M. Onorato, G. Genty, J. M. Dudley, and N. Akhmediev, Hydrodynamic Supercontinuum, Phys. Rev. Lett. 111, 054104 (2013).

[18] B. B. Frisquet, B. Kibler, and G. Millot, Collision of Akhmediev Breathers in Nonlinear Fiber Optics, Phys. Rev. X 3, 041032 (2013).

[19] J. M. Dudley, G. Genty, and S. Coen, Supercontinuum Generation in Photonic Crystal Fiber, Rev. Mod. Phys. 78, 1135 (2006).

[20] B. Barviau, B. Kibler, A. Kudlinski, A. Mussot, G. Millot, and A. Picozzi, Experimental Signature of Optical Wave Thermalization through Supercontinuum Generation in Photonic Crystal Fiber, Opt. Express 17, 7392 (2009).

[21] G. B. Whitham, Linear and Nonlinear Waves (Wiley, New York, 1974).

[22] R. H. Clarke, R. K. Smith, and D. G. Reid, The Morning Glory of the Gulf of Carpentaria: An Atmospheric Undular Bore, Mon. Weather Rev. 109, 1726 (1981); T. A. Coleman, K. R. Knupp, and D. Herzmann, The Spectacular Undular Bore in Iowa on 2 October 2007, Mon. Weather Rev. 137, 495 (2009).

[23] K. G. Lamb and Y. Liren, The Evolution of Internal Wave Undular Bores: Comparisons of a Fully Nonlinear Numerical Model with Weakly Nonlinear Theory, J. Phys. Oceanogr. 26, 2712 (1996); G. A. El, R. H. J. Grimshaw, and N. F. Smyth, Unsteady Undular Bores in Fully Nonlinear Shallow-Water Theory, Phys. Fluids 18, 027104 (2006).

[24] H. Chanson, Tidal Bores Aegir, Eagre, Mascaret, Proroca: Theory and Observations (World Scientific, Singapore, 2012).

[25] T. B. Benjamin and M. J. Lighthill, On Cnoidal Waves and Bores, Proc. R. Soc. A 224, 448 (1954); D. H. Peregrine, Calculation of the Developments of an Undular Bore, J. Fluid Mech. 25, 321 (1966); R. S. Johnson, Shallow Water Waves on a Viscous Fluid-The Undular Bore, Phys. Fluids 15, 1693 (1972).

[26] R. Z. Sagdeev, The Fine Structure of a Shock Wave Front Propagated across a Magnetic Field in a Rarefied Plasma, Sov. Phys. Tech. Phys. 6, 867 (1962).

[27] N. J. Zabusky and M. D. Kruskal, Interaction of Solitons in a Collisionless Plasma and the Recurrence of Initial States, Phys. Rev. Lett. 15, 240 (1965).

[28] R. J. Taylor, D. R. Baker, and H. Ikezi, Observation of Collisionless Electrostatic Shocks, Phys. Rev. Lett. 24, 206 (1970).

[29] A. V. Gurevich and L. P. Pitaevskii, Nonstationary structure of a collisionless shock wave, Sov. Phys. JETP 38, 291 (1974).

[30] A. V. Gurevich and A. L. Krylov, Dissipationless Shock Waves in Media with Positive Dispersion, Sov. Phys. JETP 65, 944 (1987); A. V. Gurevich, A. L. Krylov, and G. A. El, Evolution of a Riemann Wave in Dispersive Hydrodynamics, Sov. Phys. JETP 74, 957 (1992).

[31] P. D. Lax, C. D. Levermore, and S. Venakides, The Generation and Propagation of Oscillations in Dispersive Initial Value Problems and Their Limiting Behavior, in 
Important Developments in Soliton Theory, edited by A. S. Fokas and V.E. Zakharov (Springer, Berlin, 1994), p. 205.

[32] J. C. Bronski and D. W. McLaughlin, Semiclassical Behavior of the NLS Equation: Optical Shocks-Focusing Instabilities in Singular Limits of Dispersive Waves, NATO ASI Ser., Ser. B 320, 21 (1994).

[33] A. M. Kamchatnov, Nonlinear Periodic Waves and Their Modulations-An Introductory Course (World Scientific, Singapore, 2000).

[34] A. M. Kamchatnov, R. A. Kraenkel, and B. A. Umarov, Asymptotic Soliton Train Solutions of the Defocusing Nonlinear Schroödinger Equation, Phys. Rev. E 66, 036609 (2002).

[35] G. A. El, Resolution of a Shock in Hyperbolic Systems Modified by Weak Dispersion, Chaos 15, 037103 (2005).

[36] B. Dubrovin, On Hamiltonian Perturbations of Hyperbolic Systems of Conservation Laws, II: Universality of Critical Behaviour, Commun. Math. Phys. 267, 117 (2006).

[37] M. A. Hoefer and M. J. Ablowitz, Interactions of Dispersive Shock Waves, Physica (Amsterdam) 236D, 44 (2007).

[38] A. Fratalocchi, C. Conti, G. Ruocco, and S. Trillo, FreeEnergy Transition in a Gas of Noninteracting Nonlinear Wave Particles, Phys. Rev. Lett. 101, 044101 (2008).

[39] T. Grava and C. Klein, A Numerical Study of the Small Dispersion Limit of the KdV Equations and Asymptotic Solutions, Physica (Amsterdam) 241D, 2246 (2012).

[40] J. Garnier, G. Xu, S. Trillo, and A. Picozzi, Incoherent Dispersive Shocks in the Spectral Evolution of Random Waves, Phys. Rev. Lett. 111, 113902 (2013).

[41] Z. Dutton, M. Budde, C. Slowe, and L. V. Hau, Observation of Quantum Shock Waves Created with Ultra-Compressed Slow Light Pulses in a Bose-Einstein Condensate, Science 293, 663 (2001).

[42] M. A. Hoefer, M. J. Ablowitz, I. Coddington, E. A. Cornell, P. Engels, and V. Schweikhard, Dispersive and Classical Shock Waves in Bose-Einstein Condensates and Gas Dynamics, Phys. Rev. A 74, 023623 (2006).

[43] R. Meppelink, S. B. Koller, J. M. Vogels, P. van der Straten, E. D. van Ooijen, N. R. Heckenberg, H. RubinszteinDunlop, S. A. Haine, and M. J. Davis, Observation of Shock Waves in a Large Bose-Einstein Condensate, Phys. Rev. A 80, 043606 (2009).

[44] J. J. Chang, P. Engels, and M. A. Hoefer, Formation of Dispersive Shock Waves by Merging and Splitting Bose-Einstein Condensates, Phys. Rev. Lett. 101, 170404 (2008).

[45] A. Amo, S. Pigeon, D. Sanvitto, V. G. Sala, R. Hivet, I. Carusotto, F. Pisanello, G. Lemnager, R. Houdr, E. Giacobino, C. Ciuti, and A. Bramati, Polariton Superfluids Reveal Quantum Hydrodynamic Solitons, Science 332, 1167 (2011).

[46] A. M. Kamchatnov and N. Pavloff, Generation of Dispersive Shock Waves by the Flow of a Bose-Einstein Condensate Past a Narrow Obstacle, Phys. Rev. A 85, 033603 (2012).

[47] N. K. Lowman and M. A. Hoefer, Fermionic Shock Waves: Distinguishing Dissipative versus Dispersive Regularizations, Phys. Rev. A 88, 013605 (2013).
[48] S. Jia, W. Wan, and J. W. Fleischer, Dispersive Shock Waves in Nonlinear Arrays, Phys. Rev. Lett. 99, 223901 (2007).

[49] S. Jia, W. Wan, and J. W. Fleischer, Forward Four-Wave Mixing with Defocusing Nonlinearity, Opt. Lett. 32, 1668 (2007).

[50] W. Wan, S. Muenzel, and J. W. Fleischer, Wave Tunneling and Hysteresis in Nonlinear Junctions, Phys. Rev. Lett. 104, 073903 (2010).

[51] C. Conti, A. Fratalocchi, M. Peccianti, G. Ruocco, and S. Trillo, Observation of a Gradient Catastrophe Generating Solitons, Phys. Rev. Lett. 102, 083902 (2009).

[52] N. Ghofraniha, S. Gentilini, V. Folli, E. Del Re, and C. Conti, Shock Waves in Disordered Media, Phys. Rev. Lett. 109, 243902 (2012); A. Fratalocchi, A. Armaroli, and S. Trillo, Time-Reversal Focusing of an Expanding Soliton Gas in Disordered Replicas, Phys. Rev. A 83, 053846 (2011).

[53] W. J. Tomlinson, R. H. Stolen, and A. M. Johnson, Optical Wave Breaking of Pulses in Nonlinear Optical Fibers, Opt. Lett. 10, 457 (1985).

[54] D. Anderson, M. Desaix, M. Lisak, and M. L. QuiroigaTeixeiro, Wave Breaking in Nonlinear Optical Fibers, J. Opt. Soc. Am. B 9, 1358 (1992).

[55] J.E. Rothenberg and D. Grischkowsky, Observation of the Formation of an Optical Intensity Shock and Wave-Breaking in the Nonlinear Propagation of Pulses in Optical Fibers, Phys. Rev. Lett. 62, 531 (1989).

[56] J. E. Rothenberg, Colliding Visible Picosend Pulses in Optical Fibers, Opt. Lett. 15, 443 (1990); Dark Soliton Trains Formed by Visible Pulse Collisions in Optical Fibers, Opt. Commun. 82, 107 (1991); J. E. Rothenberg and H. K. Heinrich, Observation of the Formation of DarkSoliton Trains in Optical Fibers, Opt. Lett. 17, 261 (1992).

[57] J. R. Thompson and R. Roy, Nonlinear Dynamics of Multiple Four-Wave Mixing in a Single Mode Fiber, Phys. Rev. A 43, 4987 (1991).

[58] S. Trillo, S. Wabnitz, and T. A. B. Kennedy, Nonlinear Dynamics of Dual-Frequency Pumped Multiwave Mixing in Optical Fibers, Phys. Rev. A 50, 1732 (1994).

[59] M. E. Marhic, Fiber Optics Parametric Amplifiers, Oscillators, and Related Devices (Cambridge University Press, Cambridge, England, 2007).

[60] S. Trillo and A. Valiani, Hydrodynamic Instability of FourWave-Mixing, Opt. Lett. 35, 3967 (2010).

[61] R. Salem, M. A. Foster, A. C. Turner, J. E. Sharping, B. S. Schmidt, M. Lipson, and A. L. Gaeta, Broadband Optical Parametric Gain on a Silicon Photonic Chip, Nature (London) 441, 960 (2006).

[62] M. Ferrera, L. Razzari, D. Duchesne, R. Morandotti, Z. Yang, M. Liscidini, J. E. Sipe, S. Chu, B. E. Little, and D. J. Moss, Low-Power Continuous-Wave Nonlinear Optics in Doped Silica Glass Integrated Waveguide Structures, Nat. Photonics 2, 737 (2008).

[63] X. Liu, J. R. M. Osgood, Y. A. Vlasov, and W. M. J. Green, Mid-infrared Optical Parametric Amplifier Using Silicon Nanophotonic Waveguides, Nat. Photonics 4, 557 (2010).

[64] F. Morichetti, A. Canciamilla, C. Ferrari, A. Samarelli, M. Sorel, and A. Melloni, Travelling-Wave Resonant Four-Wave Mixing Breaks the Limits of Cavity-Enhanced All-Optical Wavelength Conversion, Nat. Commun. 2, 296 (2011). 
[65] X. Liu, B. Kuyken, G. Roelkens, R. Baets, R. M. Osgood, Jr., and W. M. J. Green, Bridging the Mid-infrared-toTelecom Gap with Silicon Nanophotonic Spectral Translation, Nat. Photonics 6, 667 (2012).

[66] J. Fatome, S. Pitois, and G. Millot, 20-GHz-to-1-Thz Repetition Rate Pulse Sources Based on Multiple FourWave-Mixing in Optical Fibers, IEEE J. Quantum Electron. 42, 1038 (2006).

[67] R. Salem, M. A. Foster, A. C. Turner, D. F. Geraghty, M. Lipson, and A. L. Gaeta, Signal Regeneration Using LowPower Four-Wave Mixing on Silicon Chip, Nat. Photonics 2, 35 (2007).

[68] L. Razzari, D. Duchesne, M. Ferrera, R. Morandotti, S. Chu, B. E. Little, and D. J. Moss, CMOS-Compatible Integrated Optical Hyper-parametric Oscillator, Nat. Photonics 4, 41 (2009).

[69] M. Peccianti, A. Pasquazi, Y. Park, B. E. Little, S. T. Chu, D. J. Moss, and R. Morandotti, Demonstration of a Stable Ultrafast Laser Based on a Nonlinear Microcavity, Nat. Commun. 3, 765 (2012).

[70] Z. Tong, C. Lundström, P. A. Andrekson, C. J. McKinstrie, M. Karlsson, D. J. Blessing, E. Tipsuwannakul, B. J. Puttnam, H. Toda, and L. Grüner-Nielsen, Towards Ultrasensitive Optical Links Enabled by Low-Noise Phase-Sensitive Amplifiers, Nat. Photonics 5, 430 (2011).

[71] T. J. Kippenberg, R. Holzwarth, and S. A. Diddams, Microresonator-Based Optical Frequency Combs, Science 332, 555 (2011).

[72] F. Ferdous, H. Miao, D. E. Leaird, K. Srinivasan, J. Wang, L. Chen, L. T. Varghese, and A. M. Weiner, Spectral Line-by-Line Pulse Shaping of on-Chip Microresonator Frequency Combs, Nat. Photonics 5, 770 (2011).

[73] M. Erkintalo, Y. Q. Xu, S. G. Murdoch, J. M. Dudley, and G. Genty, Cascaded Phase Matching and Nonlinear Symmetry Breaking in Fiber Frequency Combs, Phys. Rev. Lett. 109, 223904 (2012).

[74] Z. Tong, A. O. J. Wiberg, E. Myslivets, B. P. P. Kuo, N. Alic, and S. Radic, Spectral Linewidth Preservation in Parametric Frequency Combs Seeded by Dual Pumps, Opt. Express 20, 17610 (2012).

[75] J. Fatome, C. Finot, A. Armaroli, and S. Trillo, Observation of Modulationally Unstable Multi-wave Mixing, Opt. Lett. 38, 181 (2013); A. Armaroli and S. Trillo, Collective
Modulational Instability in Multiple Four-Wave Mixing, Opt. Lett. 36, 1999 (2011).

[76] S. Arismar Cerqueira, Jr., J. M. Chavez Boggio, A. A. Rieznik, H. E. Hernandez-Figueroa, H. L. Fragnito, and J. C. Knight, Highly Efficient Generation of Broadband Cascaded Four-Wave Mixing Products, Opt. Express 16, 2816 (2008).

[77] J. D. Harvey, R. Leonhardt, S. Coen, G. K. L. Wong, J. C. Knight, W. J. Wadsworth, and P. S. J. Russell, Scalar Modulation Instability in the Normal Dispersion Regime by Use of a Photonic Crystal Fiber, Opt. Lett. 28, 2225 (2003).

[78] C. J. McKinstrie and M. G. Raymer, Four-Wave-Mixing Cascades Near the Zero-Dispersion Frequency, Opt. Express 14, 9600 (2006).

[79] M. Erkintalo, K. Hammani, B. Kibler, C. Finot, N. Akhmediev, J. M. Dudley, and G. Genty, Higher-Order Modulation Instability in Nonlinear Fiber Optics, Phys. Rev. Lett. 107, 253901 (2011); G. Van Simaeys, P. Emplit, and M. Haelterman, Experimental Demonstration of the Fermi-Pasta-Ulam Recurrence in a Modulationally Unstable Optical Wave, Phys. Rev. Lett. 87, 033902 (2001); K. Tai, A. Hasegawa, and A. Tomita, Observation of Modulation Instability in Optical Fibers, Phys. Rev. Lett. 56, 135 (1986).

[80] See Supplemental Material at http://link.aps.org/ supplemental/10.1103/PhysRevX.4.021022 for more technical details about the normalization of the NLS equation [Eq. (2)] and the experimental setup in Fig. 3.

[81] In the regime considered here, only the standard cubic term associated with the Kerr effect is responsible for steepening and shock formation, whereas additional steepening terms, generally relevant for short pulses, are negligible; see, e.g., discussion in F. Demartini, C. H. Townes, T. K. Gustafson, and P. L. Kelley, Self-Steepening of Light Pulses, Phys. Rev. 164, 312 (1967); D. Anderson and M. Lisak, Nonlinear Asymmetric Self-Phase Modulation and Self-Steepening of Pulses in Long Optical Waveguides, Phys. Rev. A 27, 1393 (1983).

[82] C. Milian, D. V. Skryabin, and A. Ferrando, Continuum Generation by Dark Solitons, Opt. Lett. 34, 2096 (2009).

[83] See C. S. West and T. A. B. Kennedy, Optical Multiwave Mixing: Dark Solitary Wave Trains and Quasiperiodic Dynamics, Phys. Rev. A 47, 1252 (1993), and references therein. 